\title{
COURANT-SHARP EIGENVALUES OF COMPACT FLAT SURFACES: KLEIN BOTTLES AND CYLINDERS
}

\author{
PIERRE BÉRARD, BERNARD HELFFER, AND ROLA KIWAN
}

\begin{abstract}
The question of determining for which eigenvalues there exists an eigenfunction which has the same number of nodal domains as the label of the associated eigenvalue (Courant-sharp property) was motivated by the analysis of minimal spectral partitions. In previous works, many examples have been analyzed corresponding to squares, rectangles, disks, triangles, tori, Möbius strips,.... A natural toy model for further investigations is the flat Klein bottle, a nonorientable surface with Euler characteristic 0, and particularly the Klein bottle associated with the square torus, whose eigenvalues have higher multiplicities. In this note, we prove that the only Courant-sharp eigenvalues of the flat Klein bottle associated with the square torus (resp. with square fundamental domain) are the first and second eigenvalues. We also consider the flat cylinders $(0, \pi) \times \mathbb{S}_{r}^{1}$ where $r \in\{0.5,1\}$ is the radius of the circle $\mathbb{S}_{r}^{1}$, and we show that the only Courant-sharp Dirichlet eigenvalues of these cylinders are the first and second eigenvalues.
\end{abstract}

\section{INTRODUCTION}

Given a compact Riemannian surface $(M, g)$, we write the eigenvalues of the LaplaceBeltrami operator $-\Delta_{g}$,

$$
\lambda_{1}(M)<\lambda_{2}(M) \leq \lambda_{3}(M) \leq \ldots,
$$

in nondecreasing order, starting from the label 1, with multiplicities accounted for. If the boundary $\partial M$ of $M$ is non-empty, we consider Dirichlet eigenvalues.

Courant's nodal domain theorem (1923) states that any eigenfunction associated with the eigenvalue $\lambda_{k}$ has at most $k$ nodal domains (connected components of the complement of the zero set of $u$ ). The eigenvalue $\lambda_{k}$ is called Courant-sharp if there exists an associated eigenfunction with precisely $k$ nodal domains. It follows from Courant's theorem that the eigenvalues $\lambda_{1}$ and $\lambda_{2}$ are Courant-sharp, and that $\lambda_{k-1}<\lambda_{k}$ whenever $\lambda_{k}$ is Courant-sharp. Courant-sharp eigenvalues appear naturally in the context of partitions, [11].

Back in 1956, Pleijel proved that there are only finitely many Courant-sharp eigenvalues. More precisely, Pleijel's original proof [24] applied to Dirichlet eigenvalues of bounded domains in $\mathbb{R}^{2}$. It was later adapted to more general domains $([23])$ and to general closed Riemannian manifolds ([6]).

Courant-sharp eigenvalues of flat tori and Möbius strips were studied in $[13,19,4]$ and [5] respectively. In view of the classification of complete, flat surfaces (see [18, p. 222-223], or [26, Chap. 2.5]) it is natural to investigate the Courant-sharp property

Date: April 13, 2021 (BHK-klein-cylinder-210408.tex).

2010 Mathematics Subject Classification. 58C40, 49Q10.

Key words and phrases. Spectral theory, Courant theorem, Laplacian, Nodal sets, Klein bottle, Cylinder.

The authors would like to thank the referee for his comments. 
for the other compact flat surfaces, Klein bottles and cylinders. The purpose of the present paper is to prove the following theorems.

Theorem 1.1. Let $\mathbb{K}_{1}$ denote the flat Klein bottle associated with the square torus, resp. $\mathbb{K}_{2}$ the flat Klein bottle whose fundamental domain is a square. Then the only Courant-sharp eigenvalues of $\mathbb{K}_{c}, c \in\{1,2\}$, are $\lambda_{1}$ and $\lambda_{2}$.

Theorem 1.2. Let $\mathcal{C}_{r}$ denote the flat cylinder $(0, \pi) \times \mathbb{S}_{r}^{1}$, with the product metric. Here $\mathbb{S}_{r}^{1}$ denotes the circle with radius $r$. Then, for $r \in\left\{\frac{1}{2}, 1\right\}$, the only Courantsharp Dirichlet eigenvalues of $\mathcal{C}_{r}$, are $\lambda_{1}$ and $\lambda_{2}$.

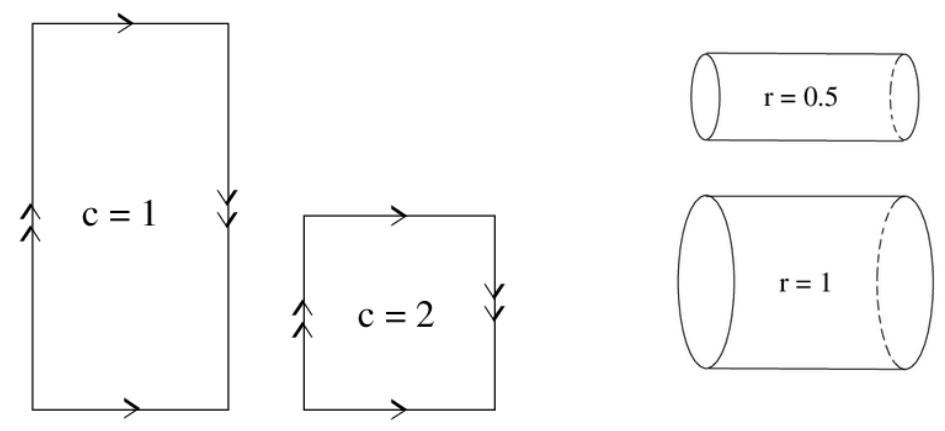

FiguRE 1.1. The Klein bottles $\mathbb{K}_{c}$ (left) and the cylinders $\mathcal{C}_{r}$ (right).

Courant-sharp eigenvalues have previously been determined for several compact surfaces. We refer to the following papers and their bibliographies.

$\diamond$ Closed surfaces: round 2-sphere and projective plane, [22]; flat tori, [13, 19, 4].

$\diamond$ Plane domains and compact surfaces with boundary (different boundary conditions might be considered): square, [24, 3, 16, 10, 9]; equilateral triangle, [4]; 2-rep-tiles, [1]; thin cylinders, [12]; Möbius strips, [5].

There are also a few results in higher dimensions, see for example [15, 20, 14].

Most of the papers mentioned above adapt the method introduced by Pleijel in [24] to the example at hand.

The paper is organized as follows. In Section 2, we recall the main lines of Pleijel's method. More precisely, we show how a lower bound on the ratio $\frac{\lambda_{k}(M)}{k}$ and a lower bound on the Weyl counting function can be used to restrict the search for Courantsharp eigenvalues to a finite set of eigenvalues. To actually determine the Courantsharp eigenvalues one then needs to analyze the nodal patterns of eigenfunctions in a finite set of eigenspaces. In Section 3, we recall some basic facts concerning Klein bottles. In Sections 4 and 5, we adapt Pleijel's method (Section 2) to the flat Klein bottles $\mathbb{K}_{1}$ and $\mathbb{K}_{2}$. In Section 6, we adapt Pleijel's method (Section 2) to the flat cylinders $\mathcal{C}_{r}$, with $r \in\left\{\frac{1}{2}, 1\right\}$.

\section{Pleijel's method Summarized}

In order to prove that the number of Courant-sharp (Dirichlet) eigenvalues of a compact Riemannian surface $(M, g)$ is finite, one only needs two ingredients, 
(1) the classical Weyl asymptotic law, $W_{M}(\lambda)=\frac{|M|}{4 \pi} \lambda+O(\sqrt{\lambda})$, for the counting function $W_{M}(\lambda)=\left\{j \mid \lambda_{j}(M)<\lambda\right\}$, where $|M|$ denotes the area of $M$;

(2) an inequality à la Faber-Krahn for domains $\omega \subset M$ with small enough area,

$$
\delta_{1}(\omega) \geq(1-\varepsilon)^{2} \frac{\pi j_{0,1}^{2}}{|\omega|} \text { provided that }|\omega| \leq C(M, \varepsilon)|M|
$$

for some $\varepsilon \in[0,1)$, and for some constant $C(M, \varepsilon)$. Here, $\delta_{1}(\omega)$ denotes the least Dirichlet eigenvalue of $\omega$, and $j_{0,1}$ is the first positive zero of the Bessel function $J_{0}\left(j_{0,1} \approx 2.404825\right)$.

Note that the right-hand side of $(2.1)$ is equal to $(1-\varepsilon)^{2} \delta_{1}\left(\omega^{*}\right)$, where $\omega^{*}$ denotes a disk in $\mathbb{R}^{2}$ with area equal to $|\omega|$. The existence of such an inequality (for a given $\varepsilon \in(0,1))$ follows from the asymptotic isoperimetric inequality proved in [6, Appendice C], and from the usual symmetrization argument to translate the isoperimetric inequality into a Faber-Krahn type inequality for the first Dirichlet eigenvalue, [6, Lemme 15].

In order to give more quantitative information on the Courant-sharp eigenvalues, one needs a lower bound on the Weyl function, in the form,

$$
W_{M}(\lambda) \geq \frac{|M|}{4 \pi} \lambda-A(M) \sqrt{\lambda}-B(M), \text { for all } \lambda \geq 0
$$

for some constants $A(M)$ and $B(M)$ depending on the geometry of $(M, g)$.

Lemma 2.1. Assume that $(M, g)$ satisfies (2.1), with $\varepsilon<1-\frac{2}{j_{0,1}}$, and (2.2). Let $\lambda_{k}(M)$ be a Courant-sharp eigenvalue with $k \geq \frac{1}{C(M, \varepsilon)}$. Then,

$$
\begin{gathered}
\frac{\lambda_{k}(M)}{k} \geq(1-\varepsilon)^{2} \frac{\pi j_{0,1}^{2}}{|M|}, \\
\frac{|M|}{(1-\varepsilon)^{2} \pi j_{0,1}^{2}} \lambda_{k}(M) \geq k \geq \frac{|M|}{4 \pi} \lambda_{k}(M)-A(M) \sqrt{\lambda_{k}(M)}-B(M)+1,
\end{gathered}
$$

In particular, $F_{M, \varepsilon}\left(\lambda_{k}(M)\right) \leq 0$, where

$$
F_{M, \varepsilon}(\lambda)=\frac{|M|}{4 \pi}\left(1-\frac{4}{(1-\varepsilon)^{2} j_{0,1}^{2}}\right) \lambda-A(M) \sqrt{\lambda}-B(M)+1,
$$

so that $\sqrt{\lambda_{k}(M)} \leq D(M, \varepsilon)$, where $D(M, \varepsilon)$ is the largest root of the equation $F_{M, \varepsilon}(\lambda)=0$.

Proof. To prove (2.3), choose any eigenfunction associated with $\lambda_{k}(M)$ and having precisely $k$ nodal domains. Since $k \geq \frac{1}{C(M, \varepsilon)}$, one of them, call it $\omega$, has area $|\omega| \leq \frac{|M|}{k} \leq C(M, \varepsilon)|M|$, so that its first Dirichlet eigenvalue satisfies (2.1). Then, use the fact that $\lambda_{k}(M)=\delta_{1}(\omega)$.

To prove (2.4), use the fact that $k-1=W_{M}\left(\lambda_{k}(M)\right)$ (this is because the eigenvalue is Courant-sharp), and apply inequalities (2.2) and (2.3).

Since $j_{0,1}>2$, choosing $\varepsilon<1-\frac{2}{j_{0,1}}$, the coefficient of the leading term in $F_{M, \varepsilon}$ is positive, and the function tends to infinity when $\lambda$ tends to infinity. 
Corollary 2.2. For $\varepsilon<1-\frac{2}{j_{0.1}}$, in order to be Courant-sharp, the eigenvalue $\lambda_{k}(M)$ must satisfy $\lambda_{k-1}(M)<\lambda_{k}(M)$, and

$$
\left\{\begin{array}{l}
\text { either } k<\frac{1}{C(M, \varepsilon)}, \\
\text { or } k \geq \frac{1}{C(M, \varepsilon)}, \sqrt{\lambda_{k}(M)} \leq D(M, \varepsilon), \text { and } \frac{\lambda_{k}(M)}{k} \geq(1-\varepsilon)^{2} \frac{\pi j_{0,1}^{2}}{|M|} .
\end{array}\right.
$$

To conclude whether the eigenvalue $\lambda_{k}(M)$ is actually Courant-sharp, it remains to determine the maximum number of nodal domains of an eigenfunction in the eigenspace $\mathcal{E}\left(\lambda_{k}(M)\right)$.

\section{Remarks 2.3.}

(1) In Sections 4 and 6, we will use the fact that we can choose $\varepsilon=0$ in the isoperimetric inequality (2.1) for the flat Klein bottles and for the flat cylinders. This follows from [17], and was already used in [19, 4, 5] for the flat torus and for the Möbius band.

(2) When $\partial M \neq \emptyset$, and for Neumann or Robin eigenfunctions of $(M, g)$, the inequality (2.1) can only be applied to a nodal domain which does not touch the boundary $\partial M$. To prove a result à la Pleijel for Neumann or Robin eigenfunctions, as in $[25,21,16,10,9]$, it is necessary to take care of this difficulty.

(3) The proof of Lemma 2.1 also yields the following inequality. Let $\kappa(k)$ denote the maximal number of nodal domains of an eigenfunction associated with $\lambda_{k}$. Then,

$$
\limsup _{k} \frac{\kappa(k)}{k} \leq \gamma(2):=\left(\frac{2}{j_{0,1}}\right)^{2}<1 .
$$

This inequality, which generalizes Pleijel's inequality [24] valid for plane domains, is actually a particular case of a general result valid for any closed Riemannian manifold, [6]. The interesting feature is that the upper bound $\gamma(n)<1$ only depends on the dimension.

\section{Preliminaries on Klein Bottles}

3.1. Klein bottles. In this note, we are interested in the flat Klein bottles. More precisely, given $a, b>0$, we consider the isometries of $\mathbb{R}^{2}$ given by

$$
\left\{\begin{array}{l}
\tau_{1}:(x, y) \mapsto(x, y+b), \\
\tau_{2}:(x, y) \mapsto(x+a, y), \\
\tau:(x, y) \mapsto\left(x+\frac{a}{2}, b-y\right) .
\end{array}\right.
$$

We denote by $G_{2}$ (resp. $G$ ) the group generated by $\tau_{1}$ and $\tau_{2}$ (resp. by $\tau_{1}$ and $\tau$ ). These groups act properly and freely by isometries on $\mathbb{R}^{2}$ equipped with the usual scalar product. Since $\tau^{2}=\tau_{2}$, the group $G_{2}$ is a subgroup of index 2 of the group $G$. We denote by $\mathbb{T}_{a, b}$ (resp. $\mathbb{K}_{a, b}$ ) the torus $\mathbb{R}^{2} / G_{2}$ (resp. the Klein bottle $\mathbb{R}^{2} / G$ ). We equip $\mathbb{T}_{a, b}$ and $\mathbb{K}_{a, b}$ with the induced flat Riemannian metrics.

A fundamental domain for the action of $G_{2}$ (resp. $\left.G\right)$ on $\mathbb{R}^{2}$ is the rectangle $\mathcal{T}_{a, b}=$ $(0, a) \times(0, b)\left(\right.$ resp. the rectangle $\mathcal{K}_{a, b}=\left(0, \frac{a}{2}\right) \times(0, b)$, see Figure $\left.3.1(\mathrm{~A})\right)$. The horizontal sides of $\mathcal{K}_{a, b}$ are identified with the same orientation, the vertical sides are identified with the opposite orientations. 
The geodesics of the Klein bottle are the images of the lines in $\mathbb{R}^{2}$ under the Riemannian covering map $\mathbb{R}^{2} \rightarrow \mathbb{K}_{a, b}$ (see [8]). They can be looked at in the fundamental domain $\mathcal{K}_{a, b}$, taking into account the identifications $(x, 0) \sim(x, b)$ and $(0, y) \sim\left(\frac{a}{2}, b-y\right)$. Among them, we have some special geodesics, see Figure $3.1(\mathrm{~B})$ $(\mathrm{C})$,

$\diamond t \mapsto(t, 0)$ and $t \mapsto\left(t, \frac{b}{2}\right)$, for $0 \leq t \leq \frac{a}{2}$, which are periodic geodesics of length $\frac{a}{2}$;

$\diamond$ for $0<y_{0}<\frac{b}{2}, \gamma_{y_{0}}: t \mapsto \begin{cases}\left(t, y_{0}\right), & 0 \leq t \leq \frac{a}{2}, \\ \left(t-\frac{a}{2}, b-y_{0}\right), & \frac{a}{2} \leq t \leq a,\end{cases}$

which is a periodic geodesic of length $a$; the two horizontal lines in blue in Figure $3.1(\mathrm{C})$ yield a periodic geodesic of the Klein bottle;

$\diamond$ for $0 \leq x_{0} \leq \frac{a}{2}, t \mapsto\left(x_{0}, t\right)$, with $0 \leq t \leq b$, is a periodic geodesic of length $b$.

Remark 3.1. The description of geodesics of the Klein bottles as projected lines implies that the shortest, nontrivial, periodic geodesic of $\mathbb{K}_{a, b}$ has length $\min \left\{\frac{a}{2}, b\right\}$.

Remark 3.2. Scissoring the Klein bottle along the blue geodesic $t \mapsto \gamma_{y_{0}}(t)$, with $0<y_{0}<\frac{b}{2}$ and $0 \leq t \leq a$, divides the surface into two Möbius strips whose center lines are the geodesics $t \mapsto(t, 0)$ and $t \mapsto\left(t, \frac{b}{2}\right)$, see Figure $3.1(D)$.

The isometry $\tau$ of $\mathbb{R}^{2}$ induces an isometry on the torus $\mathbb{T}_{a, b}$ so that we can identify $\mathbb{K}_{a, b}$ with the quotient $\mathbb{T}_{a, b} /\{I d, \tau\}$. It follows that the eigenfunctions of the Klein bottle $\mathbb{K}_{a, b}$ are precisely the eigenfunctions of the torus $\mathbb{T}_{a, b}$ which are invariant under the map $\tau$. Because $\tau$ is orientation reversing, the surface $\mathbb{K}_{a, b}$ is non-orientable with orientation double cover $\mathbb{T}_{a, b}$.

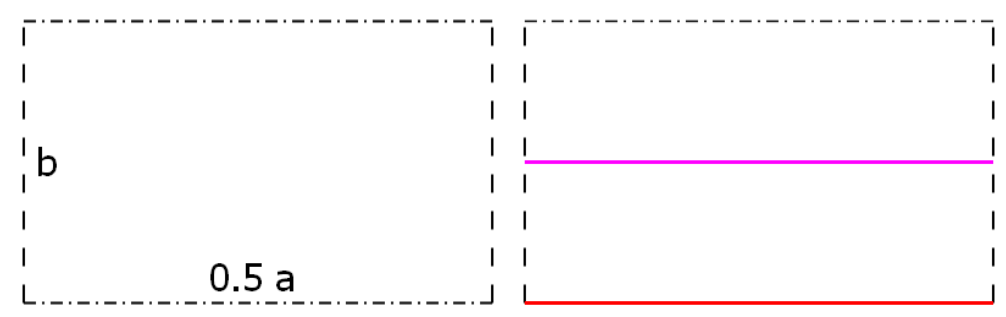

(A)

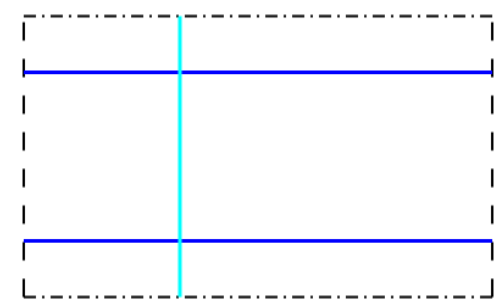

(C)
(B)

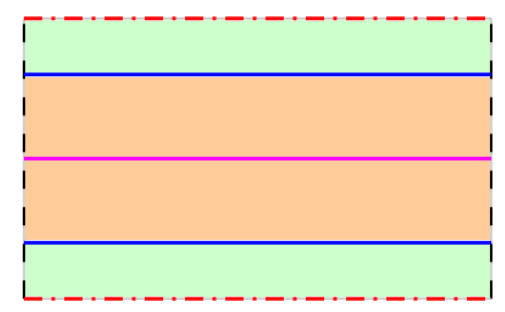

(D)

FiguRE 3.1. Fundamental domain, geodesics, partition into Möbius strips 
3.2. The spectrum of Klein bottles. A complete family of (complex) eigenfunctions of the flat torus $\mathbb{T}_{a, b}$ is

$$
f_{m, n}(x, y)=\exp \left(i \frac{2 \pi m x}{a}\right) \exp \left(i \frac{2 \pi n y}{b}\right), m, n \in \mathbb{Z},
$$

with associated eigenvalues $\hat{\lambda}(m, n)=4 \pi^{2}\left(\frac{m^{2}}{a^{2}}+\frac{n^{2}}{b^{2}}\right)$. Given some eigenvalue $\lambda$ of $\mathbb{T}_{a, b}$, we introduce the set,

$$
\mathcal{L}_{\lambda}=\left\{(m, n) \in \mathbb{Z}^{2} \mid \hat{\lambda}(m, n)=\lambda\right\}
$$

A general (complex) eigenfunction of $\mathbb{T}_{a, b}$, with eigenvalue $\lambda$, is of the form

$$
\phi=\sum_{(m, n) \in \mathcal{L}_{\lambda}} \alpha_{m, n} f_{m, n},
$$

with $\alpha_{m, n} \in \mathbb{C}$.

The function $\phi$ in invariant under $\tau, \phi=\phi \circ \tau$, if and only if

$$
\sum_{(m, n) \in \mathcal{L}_{\lambda}} \alpha_{m, n} f_{m, n}(x, y)=\sum_{(m, n) \in \mathcal{L}_{\lambda}} \alpha_{m, n}(-1)^{m} f_{m,-n}(x, y),
$$

or, equivalently, if and only if

$$
\alpha_{m,-n}=(-1)^{m} \alpha_{m, n}, \quad \forall(m, n) \in \mathcal{L}_{\lambda} .
$$

We can rewrite a $\tau$-invariant eigenfunction $\phi$ as,

$$
\phi=\sum_{(m, 0) \in \mathcal{L}_{\lambda}, m \text { even }} \alpha_{m, 0} f_{m, 0}+\sum_{(m, n) \in \mathcal{L}_{\lambda}, n>0} \alpha_{m, n}\left(f_{m, n}+(-1)^{m} f_{m,-n}\right) .
$$

The following lemma follows readily.

Lemma $3.3([7])$. A complete family of real eigenfunctions of the flat Klein bottle $\mathbb{K}_{a, b}$ is given by the following functions.

$$
\begin{cases}\text { For } m=0, n \in \mathbb{N}: & \cos \left(\frac{2 \pi n y}{b}\right) ; \\ \text { for } m \in \mathbb{N} \bullet \text { even, } n \in \mathbb{N}: & \cos \left(\frac{2 \pi m x}{a}\right) \cos \left(\frac{2 \pi n y}{b}\right) ; \sin \left(\frac{2 \pi m x}{a}\right) \cos \left(\frac{2 \pi n y}{b}\right) \\ \text { for } m \in \mathbb{N}^{\bullet} \text { odd, } n \in \mathbb{N} \bullet & \cos \left(\frac{2 \pi m x}{a}\right) \sin \left(\frac{2 \pi n y}{b}\right) ; \sin \left(\frac{2 \pi m x}{a}\right) \sin \left(\frac{2 \pi n y}{b}\right)\end{cases}
$$

Here, $\mathbb{N}$ denotes the set of non-negative integers, and $\mathbb{N}^{\bullet}$ the set of positive integers.

Remark 3.4. If $\mathcal{L}_{\lambda} \cap(\{0\} \times \mathbb{Z}) \neq \emptyset$, the multiplicity of $\lambda$ is odd; if $\mathcal{L}_{\lambda} \cap(\{0\} \times \mathbb{Z})=\emptyset$, the multiplicity of $\lambda$ is even.

3.3. Choices for $a$ and $b$. In this paper, we restrict our attention to the case $a=b=2 \pi$, i.e. to the flat Klein bottle $\mathbb{K}_{1}:=\mathbb{K}_{2 \pi, 2 \pi}$, whose fundamental domain is the rectangle $\mathcal{K}_{1}=(0, \pi) \times(0,2 \pi)$, and to the case $a=2 \pi, b=\pi$, i.e., to the flat Klein bottle $\mathbb{K}_{2}:=\mathbb{K}_{2 \pi, \pi}$, whose fundamental domain is the square $\mathcal{K}_{2}=(0, \pi) \times(0, \pi)$. As in [13] for flat tori, we could consider other values of the pair $(a, b)$, in particular we could look at what happens when $a$ is fixed, $b$ tends to zero, and vice-versa.

We denote the associated square flat tori by $\mathbb{T}_{1}$ and $\mathbb{T}_{2}$ respectively, with corresponding fundamental domains $\mathcal{T}_{1}=(0,2 \pi) \times(0,2 \pi)$ and $\mathcal{T}_{2}=(0,2 \pi) \times(0, \pi)$.

As points of the spectrum, the eigenvalues of the flat Klein bottle $\mathbb{K}_{c}, c \in\{1,2\}$ are the numbers of the form $\hat{\lambda}(p, q)=p^{2}+c^{2} q^{2}$, with $p, q \in \mathbb{N}$, and the extra condition 
that $p$ is even when $q=0$. As usual, the eigenvalues of $\mathbb{K}_{c}$ are listed in nondecreasing order, multiplicities accounted for, starting from the label 1,

$$
0=\lambda_{1}\left(\mathbb{K}_{c}\right)<\lambda_{2}\left(\mathbb{K}_{c}\right) \leq \lambda_{3}\left(\mathbb{K}_{c}\right) \leq \cdots .
$$

For $\lambda \geq 0$, we introduce the Weyl counting function,

$$
W_{\mathbb{K}_{c}}(\lambda)=\#\left\{j \mid \lambda_{j}\left(\mathbb{K}_{c}\right)<\lambda\right\} .
$$

Weyl's asymptotic law tells us that

$$
W(\lambda)=\frac{\left|\mathbb{K}_{c}\right|}{4 \pi} \lambda+O(\sqrt{\lambda})=\frac{\pi}{2 c} \lambda+O(\sqrt{\lambda})
$$

where $\left|\mathbb{K}_{c}\right|$ denotes the area of the Klein bottle, namely $\left|\mathbb{K}_{c}\right|=\frac{2 \pi^{2}}{c}$.

For later purposes, we also introduce the set

$$
\mathcal{L}_{c}(\lambda):=\left\{(m, n) \in \mathbb{N}^{2} \mid m^{2}+c^{2} n^{2}<\lambda\right\}
$$

and the counting function,

$$
L_{c}(\lambda)=\#\left(\left\{(m, n) \in \mathbb{N}^{2} \mid m^{2}+c^{2} n^{2}<\lambda\right\}\right) .
$$

\section{Courant-Sharp eigenvalues of the Klein Bottles $\mathbb{K}_{c}, c \in\{1,2\}$}

The purpose of this section is to determine the Courant-sharp eigenvalues of the Klein bottles $\mathbb{K}_{c}$, following Pleijel's method, Section 2.

Lemma 4.1. Let $\mathbb{K}_{c}, c \in\{1,2\}$ be the flat Klein bottles introduced in Section 3. Let $\lambda_{k}\left(\mathbb{K}_{c}\right)$ be a Courant-sharp eigenvalue of $\mathbb{K}_{c}$, with label $k \geq \frac{2 \pi}{c}$ (i.e. $k \geq 7$ when $c=1 ; k \geq 4$ when $c=2$ ).

When $c=1$,

$$
\begin{aligned}
\frac{\lambda_{k}\left(\mathbb{K}_{1}\right)}{k} & \geq \frac{j_{0,1}^{2}}{2 \pi} \geq 0.920422 \\
\frac{2 \pi}{j_{0,1}^{2}} \lambda_{k}\left(\mathbb{K}_{1}\right) \geq k & \geq \frac{\pi}{2} \lambda_{k}\left(\mathbb{K}_{1}\right)-2 \sqrt{\lambda_{k}\left(\mathbb{K}_{1}\right)}-2 .
\end{aligned}
$$

When $c=2$,

$$
\begin{aligned}
\frac{\lambda_{k}\left(\mathbb{K}_{2}\right)}{k} & \geq \frac{j_{0,1}^{2}}{\pi} \geq 1.840844 \\
\frac{\pi}{j_{0,1}^{2}} \lambda_{k}\left(\mathbb{K}_{2}\right) \geq k & \geq \frac{\pi}{4} \lambda_{k}\left(\mathbb{K}_{1}\right)-\frac{3}{2} \sqrt{\lambda_{k}\left(\mathbb{K}_{2}\right)}-1 .
\end{aligned}
$$

In particular, $\lambda_{k}\left(\mathbb{K}_{1}\right)<25$ and $\lambda_{k}\left(\mathbb{K}_{2}\right) \leq 47$.

Proof. By Remark 3.1, the shortest closed geodesic of $\mathbb{K}_{c}, c \in\{1,2\}$, has length $\pi$. According to $[17, \S 7]$, any domain $\omega \subset \mathbb{K}_{c}$, with area $|\omega| \leq \pi$, satisfies the Euclidean isoperimetric inequality $|\partial \omega|^{2} \geq 4 \pi|\omega|$ (where $|\partial \omega|$ denotes the length of the boundary $\partial \omega)$. It follows that

$$
\delta_{1}(\omega) \geq \frac{\pi j_{0,1}^{2}}{|\omega|} .
$$


If $\lambda_{k}\left(\mathbb{K}_{c}\right)$ is Courant-sharp with $k \geq \frac{2 \pi}{c}$, then, for any associated eigenfunction $u$ with precisely $k$ nodal domains, there exists at least one nodal domain $\omega$ with $|\omega| \leq \frac{\mathbb{K}_{c}}{k} \leq \pi$, and we have

$$
\lambda_{k}\left(\mathbb{K}_{c}\right)=\delta_{1}(\omega) \geq \frac{\pi j_{0,1}^{2}}{|\omega|} \geq k \frac{c j_{0,1}^{2}}{2 \pi} .
$$

This proves inequalities (4.1) and (4.3).

To prove the other inequalities, consider the set

$$
\mathcal{E}_{c}(\lambda):=\left\{(x, y) \mid 0 \leq x, y, x^{2}+c^{2} y^{2}<\lambda\right\} .
$$

Then,

$$
\mathcal{E}_{c}(\lambda) \subset \bigcup_{(m, n) \in \mathcal{L}_{c}(\lambda)}[m, m+1] \times[n, n+1]
$$

and hence

$$
L_{c}(\lambda)=\#\left(\mathcal{L}_{c}(\lambda)\right) \geq\left|\mathcal{E}_{c}(\lambda)\right|=\frac{\pi}{4 c} \lambda .
$$

From the description of the spectrum of $\mathbb{K}_{c}$ (Lemma 3.3), the contribution of a pair $(m, n) \in \mathcal{L}_{c}(\lambda)$, to $W_{\mathbb{K}_{c}}(\lambda)$ is
a) 2 if $m, n \geq 1$;
b) 1 if $m=0$, and $n \geq 0$;
c) 2 if $m \geq 2$ is even, and $n=0$;
d) 0 otherwise.

Recall that multiplicities also arise from the number of solutions of the equation $m^{2}+c^{2} n^{2}=m_{0}^{2}+c^{2} n_{0}^{2}$. It follows that,

$$
\begin{aligned}
W_{\mathbb{K}_{c}}(\lambda) & =2\left(L_{c}(\lambda)-\lfloor\sqrt{\lambda}\rfloor-\left\lfloor\frac{\sqrt{\lambda}}{c}\right\rfloor-1\right)+\left(\left\lfloor\frac{\sqrt{\lambda}}{c}\right\rfloor+1\right)+2\left\lfloor\frac{\sqrt{\lambda}}{2}\right\rfloor, \\
& =2 L_{c}(\lambda)-2\lfloor\sqrt{\lambda}\rfloor-\left\lfloor\frac{\sqrt{\lambda}}{c}\right\rfloor+2\left\lfloor\frac{\sqrt{\lambda}}{2}\right\rfloor-1,
\end{aligned}
$$

where $\lfloor x\rfloor$ denotes the integer part of $x \geq 0$. It follows that, for all $\lambda \geq 0$,

$$
W_{\mathbb{K}_{c}}(\lambda) \geq\left\{\begin{array}{l}
\frac{\pi}{2} \lambda-2 \sqrt{\lambda}-3 \text { when } c=1, \\
\frac{\pi}{4} \lambda-\frac{3}{2} \sqrt{\lambda}-2 \text { when } c=2 .
\end{array}\right.
$$

Inequalities (4.2) and (4.4) follow from the previous inequalities.

Table 4.1 displays the eigenvalues of $\mathbb{K}_{c}$ less than or equal to $25(c=1)$, resp. 47 $(c=2)$, the corresponding labeled eigenvalues, and the ratio $\frac{\lambda_{k_{\min }}\left(\mathbb{K}_{c}\right)}{k_{\min }}$ which should be larger than or equal to

$$
\begin{array}{ll}
\frac{j_{0,1}^{2}}{2 \pi} \approx 0.92042 & \text { if } \lambda_{k_{\min }}\left(\mathbb{K}_{1}\right) \text { is Courant-sharp, } \\
\frac{j_{0,1}^{2}}{\pi} \approx 1.84084 & \text { if } \lambda_{k_{\min }}\left(\mathbb{K}_{2}\right) \text { is Courant-sharp. }
\end{array}
$$

Since the eigenvalues $\lambda_{1}\left(\mathbb{K}_{c}\right), \lambda_{2}\left(\mathbb{K}_{c}\right)$ are Courant-sharp, we conclude from Table 4.1 that Theorem 1.1 is proved in the case $c=2$. To finish the proof of the theorem 


\begin{tabular}{|c|c|c|c|}
\hline $\mathbb{K}_{1}$ & $\lambda_{k_{\min }}\left(\mathbb{K}_{1}\right)$ & $\lambda_{k_{\max }}\left(\mathbb{K}_{1}\right)$ & $\frac{\lambda_{k_{\min }}\left(\mathbb{K}_{1}\right)}{k_{\min }}$ \\
\hline 1 & $\lambda_{2}$ & $\lambda_{2}$ & - \\
\hline 2 & $\lambda_{3}$ & $\lambda_{4}$ & - \\
\hline 4 & $\lambda_{5}$ & $\lambda_{7}$ & - \\
\hline 5 & $\lambda_{8}$ & $\lambda_{11}$ & 0.625 \\
\hline 8 & $\lambda_{12}$ & $\lambda_{13}$ & 0.6666 \\
\hline 9 & $\lambda_{14}$ & $\lambda_{14}$ & 0.6428 \\
\hline 10 & $\lambda_{15}$ & $\lambda_{18}$ & 0.6666 \\
\hline 13 & $\lambda_{19}$ & $\lambda_{22}$ & 0.6842 \\
\hline 16 & $\lambda_{23}$ & $\lambda_{25}$ & 0.6956 \\
\hline 17 & $\lambda_{26}$ & $\lambda_{29}$ & 0.6538 \\
\hline 18 & $\lambda_{30}$ & $\lambda_{31}$ & 0.6 \\
\hline 20 & $\lambda_{32}$ & $\lambda_{35}$ & 0.625 \\
\hline 25 & $\lambda_{36}$ & $\lambda_{40}$ & 0.6944 \\
\hline
\end{tabular}

\begin{tabular}{|c|c|c|c|}
\hline $\mathbb{K}_{2}$ & $\lambda_{k_{\min }}\left(\mathbb{K}_{2}\right)$ & $\lambda_{k_{\max }}\left(\mathbb{K}_{2}\right)$ & $\frac{\lambda_{k_{\min }}\left(\mathbb{K}_{2}\right)}{k_{\min }}$ \\
\hline 4 & $\lambda_{2}$ & $\lambda_{4}$ & - \\
\hline 5 & $\lambda_{5}$ & $\lambda_{6}$ & 1 \\
\hline 8 & $\lambda_{7}$ & $\lambda_{8}$ & 1.1429 \\
\hline 13 & $\lambda_{9}$ & $\lambda_{10}$ & 1.4444 \\
\hline 16 & $\lambda_{11}$ & $\lambda_{13}$ & 1.4545 \\
\hline 17 & $\lambda_{14}$ & $\lambda_{15}$ & 1.2143 \\
\hline 20 & $\lambda_{16}$ & $\lambda_{19}$ & 1.2500 \\
\hline 25 & $\lambda_{20}$ & $\lambda_{21}$ & 1.2500 \\
\hline 29 & $\lambda_{22}$ & $\lambda_{23}$ & 1.3182 \\
\hline 32 & $\lambda_{24}$ & $\lambda_{25}$ & 1.3333 \\
\hline 36 & $\lambda_{26}$ & $\lambda_{28}$ & 1.3846 \\
\hline 37 & $\lambda_{29}$ & $\lambda_{30}$ & 1.2759 \\
\hline 40 & $\lambda_{31}$ & $\lambda_{34}$ & 1.2903 \\
\hline 41 & $\lambda_{35}$ & $\lambda_{36}$ & 1.1714 \\
\hline 45 & $\lambda_{37}$ & $\lambda_{38}$ & 1.2162 \\
\hline 52 & $\lambda_{39}$ & $\lambda_{42}$ & 1.3333 \\
\hline
\end{tabular}

TABLE 4.1. The first eigenvalues of $\mathbb{K}_{1}$ (left) and $\mathbb{K}_{2}$ (right)

when $c=1$, it remains to investigate $\lambda_{3}\left(\mathbb{K}_{1}\right)$ and $\lambda_{5}\left(\mathbb{K}_{1}\right)$. This is done in the next section.

\section{The eigenvalues $\lambda_{3}\left(\mathbb{K}_{1}\right)$ and $\lambda_{5}\left(\mathbb{K}_{1}\right)$ are not Courant-SharP}

In order to finish the proof of Theorem 1.1 for the Klein bottle $\mathbb{K}_{1}$, we investigate the eigenvalues $\lambda_{3}\left(\mathbb{K}_{1}\right)=\hat{\lambda}(1,1)$ and $\lambda_{5}\left(\mathbb{K}_{2}\right)=\hat{\lambda}(2,0)=\hat{\lambda}(0,2)$.

5.1. The eigenvalue $\lambda_{3}\left(\mathbb{K}_{1}\right)$ is not Courant-sharp. A general eigenfunction associated with $\lambda_{3}$ has the form $(A \cos (x)+B \sin (x)) \sin (y)$. It is sufficient to look at eigenfunctions of the form $\sin (x-\alpha) \sin (y)$. These eigenfunctions have exactly two nodal domains in $\mathbb{K}_{1}$. It follows that $\lambda_{3}$ is not Courant-sharp, see Figure 5.1.

5.2. The eigenvalue $\lambda_{5}\left(\mathbb{K}_{1}\right)$ is not Courant-sharp. A general eigenfunction associated with $\lambda_{5}$ has the form $A \cos (2 x)+B \sin (2 x)+C \cos (2 y)$. Up to multiplication by a scalar, it suffices to consider the family $\cos \theta \cos (2 x-\alpha)+\sin \theta \cos (2 y)$, with $\theta \in[0, \pi)$ and $\alpha \in[0, \pi)$. Choosing the fundamental domain appropriately, we can assume that $\alpha=0$. Changing $y$ to $y+\frac{\pi}{2}$, we see that it suffices to consider $\theta \in\left[0, \frac{\pi}{2}\right]$. The nodal sets are known explicitly when $\theta=0$ or $\frac{\pi}{2}$. We now consider the family

$$
\phi_{\theta}(x, y)=\cos \theta \cos (2 x)+\sin \theta \cos (2 y), \quad \theta \in\left(0, \frac{\pi}{2}\right) .
$$




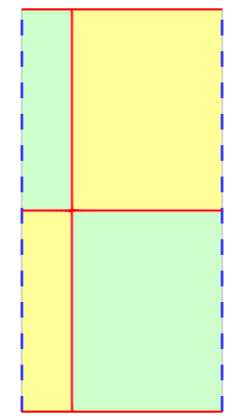

Figure 5.1. Nodal domains of $\sin (x-\alpha) \sin (y)$

The critical zeros (points at which both the function and its differential vanish) satisfy the system,

$$
\left\{\begin{array}{l}
\cos \theta \cos (2 x)+\sin \theta \cos (2 y)=0 \\
\sin (2 x)=0 \\
\sin (2 y)=0
\end{array}\right.
$$

It follows that critical zeros only occur for $\theta=\frac{\pi}{4}$, so that the nodal set of $\phi_{\theta}$ is a regular curve when $\theta \neq \frac{\pi}{4}$. The nodal set of the eigenfunction

$$
\phi_{\frac{\pi}{4}}(x, y)=\cos (2 x)+\cos (2 y)=2 \cos (x+y) \cos (x-y)
$$

is explicit, see Figure 5.2(B). An analysis à la Stern, see [5, Section 5] or [2], shows that the nodal sets of $\phi_{\theta}$ for $0<\theta<\frac{\pi}{4}$ and $\frac{\pi}{4}<\theta<\frac{\pi}{2}$ are given by Figures 5.2 (A) and $(\mathrm{C})$ respectively. More precisely, we first note that the common zeros to $\phi_{0}$ and $\phi_{\frac{\pi}{2}}$ are common zeros to all $\phi_{\theta}$. Since $\theta \in\left(0, \frac{\pi}{2}\right)$, and hence $\sin \theta \cos \theta>0$, it follows that, except for the common zeros, the nodal set of $\phi_{\theta}$ is contained in the set $\cos (2 x) \cos (2 y)<0$. Finally, depending on the sign of $\frac{\pi}{4}-\theta$, we can use the nodal set of $\cos (2 x)$ or the nodal set of $\cos (2 y)$ as barrier to obtain the behaviour described in the figures.

It follows that an eigenfunction associated with $\lambda_{5}$ has at most 4 nodal domains in $\mathbb{K}_{1}$, and hence that $\lambda_{5}\left(\mathbb{K}_{1}\right)$ is not Courant-sharp.

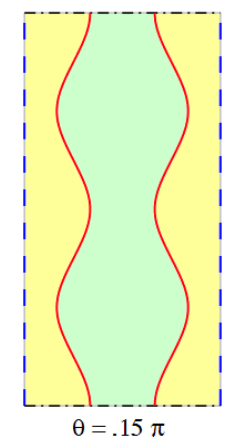

(A)

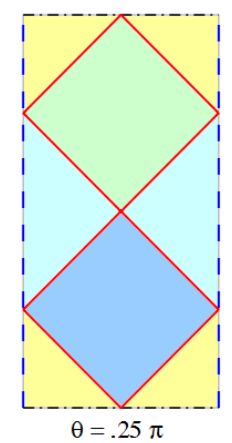

(B)

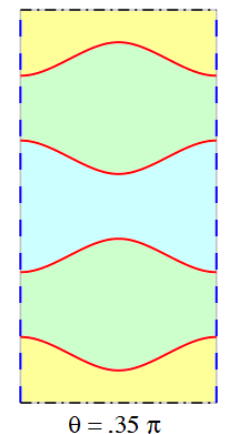

(C)

Figure 5.2. Nodal sets for $\cos \theta \cos (2 x)+\sin \theta \cos (2 y)$

This completes the proof of Theorem 1.1 in the case $c=1$. 


\section{Courant-Sharp Dirichlet eigenvalues of Flat Cylinders}

6.1. Preliminaries. Given $r>0$, let $\mathcal{C}_{r}=(0, \pi) \times \mathbb{S}_{r}^{1}$ denote the cylinder with radius $r$ and length $\pi$, equipped with the flat product metric. We consider the Dirichlet boundary condition on $\partial \mathcal{C}_{r}=\{0, \pi\} \times \mathbb{S}_{r}^{1}$. A complete family of Dirichlet eigenfunctions is given by,

$$
\begin{cases}\sin (m x) \cos \left(\frac{n y}{r}\right), & \text { for } m \in \mathbb{N}^{\bullet}, n \in \mathbb{N}, \\ \sin (m x) \sin \left(\frac{n y}{r}\right), & \text { for } m \in \mathbb{N}^{\bullet}, n \in \mathbb{N}^{\bullet},\end{cases}
$$

with associated eigenvalues the numbers $\hat{\lambda}_{r}(m, n)=m^{2}+\frac{n^{2}}{r^{2}}$.

For $m \in \mathbb{N}^{\bullet}$, the point $(m, 0)$ contributes one eigenfunction $\sin (m x)$; for $m, n \in \mathbb{N}^{\bullet}$, the point $(m, n)$ contributes two functions, $\sin (m x) \cos \left(\frac{n y}{r}\right)$ and $\sin (m x) \sin \left(\frac{n y}{r}\right)$. Multiplicities of the eigenvalues also occur when the equation $m^{2}+\frac{n^{2}}{r^{2}}=m_{0}^{2}+\frac{n_{0}^{2}}{r^{2}}$ has multiple solutions within the above range.

As usual, we arrange the Dirichlet eigenvalues of $\mathcal{C}_{r}$ in non-decreasing order, starting from the label 1, multiplicities accounted for,

$$
0<\lambda_{1}\left(\mathcal{C}_{r}\right)<\lambda_{2}\left(\mathcal{C}_{r}\right) \leq \lambda_{3}\left(\mathcal{C}_{r}\right) \leq \cdots .
$$

The purpose of this section is to prove Theorem 1.2, i.e. to determine the Courantsharp eigenvalues in two specific cases $r \in\left\{\frac{1}{2}, 1\right\}$ whose eigenvalues have higher multiplicities. The corresponding cylinder are the orientation covers of the Möbius band we studied in [5]. As in $[12,13]$, we could also consider other values of $r$, in particular $r$ close to zero or $r$ very large.

Remark 6.1. As pointed out in the introduction, $\lambda_{1}\left(\mathcal{C}_{r}\right)$ and $\lambda_{2}\left(\mathcal{C}_{r}\right)$ are always Courant-sharp, and any Courant-sharp eigenvalue $\lambda_{k}\left(\mathcal{C}_{r}\right)$ satisfies the inequality $\lambda_{k}\left(\mathcal{C}_{r}\right)>\lambda_{k-1}\left(\mathcal{C}_{r}\right)$.

Following Pleijel's method, Section 2, we introduce Weyl's counting function,

$$
W_{\mathcal{C}_{r}}(\lambda)=\#\left\{j \mid \lambda_{j}\left(\mathcal{C}_{r}\right)<\lambda\right\} .
$$

According to Weyl's law,

$$
W_{\mathcal{C}_{r}}(\lambda)=\frac{\left|\mathcal{C}_{r}\right|}{4 \pi} \lambda+O(\sqrt{\lambda})=\frac{r \pi}{2} \lambda+O(\sqrt{\lambda}),
$$

where $\left|\mathcal{C}_{r}\right|$ denotes the area of the cylinder.

6.2. Courant-sharp eigenvalues of $\mathcal{C}_{r}, r \in\left\{\frac{1}{2}, 1\right\}$. According to [17, $\left.\S 6\right]$, a domain $\omega \subset \mathcal{C}_{r}$, with area $|\omega| \leq 4 \pi r^{2}$ satisfies a Euclidean isoperimetric inequality, and hence its least Dirichlet eigenvalue satisfies

$$
\delta_{1}(\omega) \geq \frac{\pi j_{0,1}^{2}}{|\omega|}
$$

Lemma 6.2. Let $r \in\left\{\frac{1}{2}, 1\right\}$. Let $\lambda_{k}\left(\mathcal{C}_{r}\right)$ be a Courant-sharp eigenvalue of $\mathcal{C}_{r}$, with $k \geq \frac{\pi}{2 r}$. The following inequalities hold.

$$
\frac{\lambda_{k}\left(\mathcal{C}_{r}\right)}{k} \geq \frac{j_{0,1}^{2}}{2 \pi r} \geq \begin{cases}\frac{j_{0,1}^{2}}{\pi} \approx 1.840844 & \text { if } r=\frac{1}{2}, \\ \frac{j_{0,1}^{2}}{2 \pi} \approx 0.920422 & \text { if } r=1,\end{cases}
$$




$$
\begin{aligned}
& k-1=W_{\mathcal{C}_{r}}\left(\lambda_{k}\left(\mathcal{C}_{r}\right)\right) \geq \frac{\pi r}{2} \lambda_{k}\left(\mathcal{C}_{r}\right)-(2 r+1) \sqrt{\lambda_{k}\left(\mathcal{C}_{r}\right)}-2 . \\
& \text { For } r=\frac{1}{2}, \quad \frac{\pi}{4}\left(1-\frac{4}{j_{0,1}^{2}}\right) \lambda_{k}\left(\mathcal{C}_{\frac{1}{2}}\right)-2 \sqrt{\lambda_{k}\left(\mathcal{C}_{\frac{1}{2}}\right)}-1 \leq 0 \\
& \lambda_{k}\left(\mathcal{C}_{\frac{1}{2}}\right) \leq 76.25 \\
& \text { For } r=1, \quad \frac{\pi}{4}\left(1-\frac{4}{j_{0,1}^{2}}\right) \lambda_{k}\left(\mathcal{C}_{1}\right)-\frac{3}{2} \sqrt{\lambda_{k}\left(\mathcal{C}_{1}\right)}-\frac{1}{2} \leq 0 \\
& \lambda_{k}\left(\mathcal{C}_{1}\right) \leq 42.40
\end{aligned}
$$

Proof. The arguments are similar to those used in the proof of Lemma 4.1. Inequality (6.6) follows from (6.5). The inequality in (6.7) follows from the description of the Dirichlet spectrum of $\mathcal{C}_{r}$. The other inequalities follow from (6.7) and (6.6).

The following tables give the eigenvalues, the corresponding range of labels, and the ratios $\frac{\lambda_{k_{\min }}}{k_{\min }}$ for $\mathcal{C}_{\frac{1}{2}}$ (left) and $\mathcal{C}_{1}$ (right). For a Courant-sharp eigenvalue, this ratio should be greater than 1.840844 for $\mathcal{C}_{\frac{1}{2}}$, and greater than 0.920422 for $\mathcal{C}_{1}$.

According to Remark 6.1, the eigenvalues $\lambda_{1}$ and $\lambda_{2}$ are always Courant-sharp.

The table for $\mathcal{C}_{\frac{1}{2}}$ (left) shows that it only remains to analyze the eigenvalue $5=$ $\lambda_{3}\left(\mathcal{C}_{\frac{1}{2}}\right)=\lambda_{4}\left(\mathcal{C}_{\frac{1}{2}}^{2}\right)$. The associated eigenspace is generated by the eigenfunctions $\sin (x) \cos (2 y)$ and $\sin (x) \sin (2 y)$. Functions in this eigenspace have 2 nodal domains. This finishes the proof of Theorem 1.2 in the case $r=\frac{1}{2}$.

The table for $\mathcal{C}_{1}$ (right) shows that it only remains to analyze two eigenvalues 4 and 5 . The eigenvalue $4=\lambda_{4}\left(\mathcal{C}_{1}\right)$ is simple, with associated eigenfunction $\sin (2 x)$ which has two nodal domains. The eigenvalue $5=\lambda_{5}\left(\mathcal{C}_{1}\right)=\cdots=\lambda_{8}\left(\mathcal{C}_{1}\right)$ has multiplicity 4 . The corresponding eigenspace is generated by the eigenfunctions $\sin (2 x) \cos (x), \sin (2 x) \sin (x)$ and $\sin (x) \cos (2 x), \sin (x) \sin (2 y)$ which, according to [5], turn out to span the second eigenspace $\mathcal{E}\left(\lambda_{2}\left(M_{1}\right)\right)$ of the square Möbius strip, whose orientation cover is $\mathcal{C}_{1}$. Since the eigenfunctions in $\mathcal{E}\left(\lambda_{2}\left(M_{1}\right)\right)$ have two nodal domains, it follows that the eigenfunctions in the eigenspace $\mathcal{E}\left(\lambda_{5}\left(\mathcal{C}_{1}\right)\right)$ have at most four nodal domains. This finishes the proof of Theorem 1.2 in the case $r=1$. Note that one can give a precise analysis of nodal patterns in the eigenspace $\mathcal{E}\left(\lambda_{5}\left(\mathcal{C}_{1}\right)\right)$ by using the same arguments as in [5, Section 4]. 


\begin{tabular}{|c|c|c|c|}
\hline$\lambda\left(\mathcal{C}_{1 / 2}\right)$ & $k_{\min }$ & $k_{\max }$ & $\frac{\lambda_{k_{\min }}\left(\mathcal{C}_{1 / 2}\right)}{k_{\min }}$ \\
\hline 1 & 1 & 1 & - \\
\hline 4 & 2 & 2 & - \\
\hline 5 & 3 & 4 & - \\
\hline 8 & 5 & 6 & 1,60000 \\
\hline 9 & 7 & 7 & 1,28571 \\
\hline 13 & 8 & 9 & 1,62500 \\
\hline 16 & 10 & 10 & 1,60000 \\
\hline 17 & 11 & 12 & 1,54545 \\
\hline 20 & 13 & 16 & 1,53846 \\
\hline 25 & 17 & 19 & 1,47059 \\
\hline 29 & 20 & 21 & 1,45000 \\
\hline 32 & 22 & 23 & 1,45455 \\
\hline 36 & 24 & 24 & 1,50000 \\
\hline 37 & 25 & 26 & 1,48000 \\
\hline 40 & 27 & 30 & 1,48148 \\
\hline 41 & 31 & 32 & 1,32258 \\
\hline 45 & 33 & 34 & 1,36364 \\
\hline 49 & 35 & 35 & 1,40000 \\
\hline 52 & 36 & 39 & 1,44444 \\
\hline 53 & 40 & 41 & 1,32500 \\
\hline 61 & 42 & 43 & 1,45238 \\
\hline 64 & 44 & 44 & 1,45455 \\
\hline 65 & 45 & 48 & 1,44444 \\
\hline 68 & 49 & 52 & 1,38776 \\
\hline 72 & 53 & 54 & 1,35849 \\
\hline 73 & 55 & 56 & 1,32727 \\
\hline 80 & 57 & 60 & 1,40351 \\
\hline & & & \\
\hline
\end{tabular}

\begin{tabular}{|c|c|c|c|}
\hline$\lambda\left(\mathcal{C}_{1}\right)$ & $k_{\min }$ & $k_{\max }$ & $\frac{\lambda_{k_{\min }}\left(\mathcal{C}_{1}\right)}{k_{\min }}$ \\
\hline 1 & 1 & 1 & - \\
\hline 2 & 2 & 3 & 1,00000 \\
\hline 4 & 4 & 4 & 1,00000 \\
\hline 5 & 5 & 8 & 1,00000 \\
\hline 8 & 9 & 10 & 0,88889 \\
\hline 9 & 11 & 11 & 0,81818 \\
\hline 10 & 12 & 15 & 0,83333 \\
\hline 13 & 16 & 19 & 0,81250 \\
\hline 16 & 20 & 20 & 0,80000 \\
\hline 17 & 21 & 24 & 0,80952 \\
\hline 18 & 25 & 26 & 0,72000 \\
\hline 20 & 27 & 30 & 0,74074 \\
\hline 25 & 31 & 35 & 0,80645 \\
\hline 26 & 36 & 39 & 0,72222 \\
\hline 29 & 40 & 43 & 0,72500 \\
\hline 32 & 44 & 45 & 0,72727 \\
\hline 34 & 46 & 49 & 0,73913 \\
\hline 36 & 50 & 50 & 0,72000 \\
\hline 37 & 51 & 54 & 0,72549 \\
\hline 40 & 55 & 58 & 0,72727 \\
\hline 41 & 59 & 62 & 0,69492 \\
\hline 45 & 63 & 66 & 0,71429 \\
\hline 49 & 67 & 67 & 0,73134 \\
\hline 50 & 68 & 73 & 0,73529 \\
\hline & & & \\
\hline
\end{tabular}

TABLE 6.1. The first eigenvalues of $\mathcal{C}_{\frac{1}{2}}$ (left) and $\mathcal{C}_{1}$ (right)

\section{REFERENCES}

[1] Ram Band, Michael Bersudsky, and David Fajman. Courant-sharp eigenvalues of Neumann 2-rep-tiles. Lett. Math. Phys., 107(5):821-859, 2017. 2

[2] Pierre Bérard and Bernard Helffer. Nodal sets of eigenfunctions, Antonie Stern's results revisited. In Séminaire de théorie spectrale et géométrie, volume 32, pages 1-37. Université de Grenoble, 2014-2015. 10

[3] Pierre Bérard and Bernard Helffer. Dirichlet eigenfunctions of the square membrane: Courant's property, and A. Stern's and Å. Pleijel's analyses. In Analysis and geometry, volume 127 of Springer Proc. Math. Stat., pages 69-114. Springer, Cham, 2015. 2

[4] Pierre Bérard and Bernard Helffer. Courant-sharp eigenvalues for the equilateral torus, and for the equilateral triangle. Lett. Math. Phys., 106(12):1729-1789, 2016. 1, 2, 4

[5] Pierre Bérard, Bernard Helffer, and Rola Kiwan. Courant-sharp property for Dirichlet eigenfunctions on the Möbius strip. Portugaliae Mathematica, 2021. Accepted for publication. 1, 2, $4,10,11,12$

[6] Pierre Bérard and Daniel Meyer. Inégalités isopérimétriques et applications. Ann. Sci. École Norm. Sup. (4), 15(3):513-541, 1982. 1, 3, 4 
[7] Marcel Berger, Paul Gauduchon, and Edmond Mazet. Le spectre d'une variété riemannienne. Lecture Notes in Mathematics, Vol. 194. Springer-Verlag, Berlin-New York, 1971. 6

[8] Sylvestre Gallot, Dominique Hulin, and Jacques Lafontaine. Riemannian geometry. Universitext. Springer-Verlag, Berlin, third edition, 2004. 5

[9] K. Gittins and B. Helffer. Courant-sharp Robin eigenvalues for the square: the case with small Robin parameter. Ann. Math. Qué., 44(1):91-123, 2020. 2, 4

[10] Katie Gittins and Bernard Helffer. Courant-sharp Robin eigenvalues for the square and other planar domains. Port. Math., 76(1):57-100, 2019. 2, 4

[11] B. Helffer, T. Hoffmann-Ostenhof, and S. Terracini. Nodal domains and spectral minimal partitions. Ann. Inst. H. Poincaré Anal. Non Linéaire, 26(1):101-138, 2009. 1

[12] Bernard Helffer and Thomas Hoffmann-Ostenhof. Spectral minimal partitions for a thin strip on a cylinder or a thin annulus like domain with Neumann condition. In Operator methods in mathematical physics, volume 227 of Oper. Theory Adv. Appl., pages 107-115. Birkhäuser/Springer Basel AG, Basel, 2013. 2, 11

[13] Bernard Helffer and Thomas Hoffmann-Ostenhof. Minimal partitions for anisotropic tori. J. Spectr. Theory, 4(2):221-233, 2014. 1, 2, 6, 11

[14] Bernard Helffer and Rola Kiwan. Dirichlet eigenfunctions in the cube, sharpening the Courant nodal inequality. In Functional analysis and operator theory for quantum physics, EMS Ser. Congr. Rep., pages 353-371. Eur. Math. Soc., Zürich, 2017. 2

[15] Bernard Helffer and Mikael Persson Sundqvist. On nodal domains in Euclidean balls. Proc. Amer. Math. Soc., 144(11):4777-4791, 2016. 2

[16] Bernard Helffer and Mikael Persson Sundqvist. Nodal domains in the square - the Neumann case. Mosc. Math. J., 15(3):455-495, 605, 2015. 2, 4

[17] Hugh Howards, Michael Hutchings, and Frank Morgan. The isoperimetric problem on surfaces. Amer. Math. Monthly, 106(5):430-439, 1999. 4, 7, 11

[18] Shoshichi Kobayashi and Katsumi Nomizu. Foundations of differential geometry. Vol I. Interscience Publishers, a division of John Wiley \& Sons, New York-London, 1963. 1

[19] Corentin Léna. Courant-sharp eigenvalues of a two-dimensional torus. C. R. Math. Acad. Sci. Paris, 353(6):535-539, 2015. 1, 2, 4

[20] Corentin Léna. Courant-sharp eigenvalues of the three-dimensional square torus. Proc. Amer. Math. Soc., 144(9):3949-3958, 2016. 2

[21] Corentin Léna. Pleijel's nodal domain theorem for Neumann and Robin eigenfunctions. Ann. Inst. Fourier (Grenoble), 69(1):283-301, 2019. 4

[22] Josef Leydold. On the number of nodal domains of spherical harmonics. Topology, 35(2):301$321,1996.2$

[23] Jaak Peetre. A generalization of Courant's nodal domain theorem. Math. Scand., 5:15-20, 1957. 1

[24] Åke Pleijel. Remarks on Courant's nodal line theorem. Comm. Pure Appl. Math., 9:543-550, 1956. 1, 2, 4

[25] Iosif Polterovich. Pleijel's nodal domain theorem for free membranes. Proc. Amer. Math. Soc., 137(3):1021-1024, 2009. 4

[26] Joseph A. Wolf. Spaces of constant curvature. AMS Chelsea Publishing, Providence, RI, sixth edition, 2011. 1

PB: Université Grenoble Alpes and CNRS, Institut Fourier, CS 40700, F38058 Grenoble Cedex 9, France.

Email address: pierrehberard@gmail.com

BH: Laboratoire Jean Leray, Université de Nantes and CNRS, F44322 Nantes Cedex, France AND LMO (Université PARIS-SUd).

Email address: Bernard.Helffer@univ-nantes.fr

RK: American University in Dubai, P.O.Box 28282, Dubai, United Arab Emirates. Email address: rkiwan@aud.edu 